\title{
The RadioAstron Green Bank Earth Station
}

\author{
H. Alyson Ford ${ }^{a}$, Robert Anderson ${ }^{a}$, Konstantin Belousov ${ }^{b}$, Joseph J. Brandt ${ }^{a}$, \\ John M. Ford ${ }^{a}$, Boris Kanevsky ${ }^{b}$, Anatoly Kovalenko ${ }^{c}$, Yuri Y. Kovalev ${ }^{b}$, \\ Ronald J. Maddalena ${ }^{a}$, Sergei Sergeev ${ }^{d}$, Alexander Smirnov $^{b}$, \\ Galen Watts ${ }^{a}$, and Timothy Lee Weadon ${ }^{a}$ \\ ${ }^{a}$ National Radio Astronomy Observatory, P.O. Box 2, Green Bank, WV 24944, USA; \\ ${ }^{b}$ Astro Space Center of Lebedev Physical Institute, Russian Academy of Sciences, \\ Profsoyuznaya 84/32, Moscow 117997, Russia; \\ ${ }^{c}$ Pushchino Radio Astronomy Observatory, Astro Space Center of Lebedev Physical Institute, \\ Russian Academy of Sciences, Pushchino, Moscow region 142290, Russia; \\ ${ }^{d}$ Lavochkin Association, 24 Leningradskaya St., Khimki, Moscow region 141400, Russia
}

\begin{abstract}
We present the design, commissioning, and initial results of the Green Bank Earth Station (GBES), a RadioAstron data downlink station located at the National Radio Astronomy Observatory (NRAO) in Green Bank, West Virginia. The GBES uses the modernised and refurbished NRAO 140ft telescope. Antenna optics were refurbished with new motors and drives fitted to the secondary mirror positioning system, and the deformable subreflector was refurbished with a new digital controller and new actuators. A new monitor and control system was developed for the 140ft and is based on that of the Green Bank Telescope (GBT), allowing satellite tracking via a simple scheduling block. Tools were developed to automate antenna pointing during tracking. Data from the antenna control systems and logs are retained and delivered with the science and telemetry data for processing at the Astro Space Center (ASC) of the Lebedev Physical Institute (LPI) of the Russian Academy of Sciences and the mission control centre, Lavochkin Association.
\end{abstract}

Keywords: Earth Station, downlink, Space VLBI, Radio Telescope, Control Systems

\section{INTRODUCTION}

The 140-foot radio telescope (hereafter referred to as the 140ft) at the Green Bank, West Virginia site of the National Radio Astronomy Observatory (NRAO) has been refurbished and dedicated to downlinking data from the Spektr-R RadioAstron Very Long Baseline Interferometry (VLBI) satellite. The satellite, created by the Lavochkin Association, carries a $10 \mathrm{~m}$ space radio telescope that operates at frequencies ranging from 316 to $25132 \mathrm{MHz}$, covering P, L, C, and K-band. Its highly elliptical orbit extends to $370000 \mathrm{~km}$ at apogee. Observing simultaneously with several ground telescopes, Spektr-R forms the Earth's longest radio interferometer. Data from the space telescope must be collected via large Earth stations, which provide sufficient gain for signal reception at the longest distances. The initial Earth station at the Pushchino Radio Astronomy Observatory (PRAO), Russia, is limited to observing the satellite when it is above their horizon, making stations in other locations essential to maximise available downlink time. Good coverage by multiple ground stations is also required due to the lack of on-board data storage capabilities. The Green Bank Earth Station (GBES) was implemented to meet this need for geographical diversity of the Earth stations.

GBES components include an antenna, electronics to receive, process, and record signals, and system control and management hardware and software (a block diagram of the entire GBES is presented in Figure 1). The GBES uses the modernised and refurbished 140ft telescope, on which regular radio astronomy observations have been suspended since the Robert C. Byrd Green Bank Telescope (GBT) commissioning commenced in the late 1990s. In 2005, the venerable 140ft telescope was reactivated to track low Earth orbit satellites for ionospheric

Further author information: (Send correspondence to H. Alyson Ford)

H. Alyson Ford.: E-mail: aford@nrao.edu, Telephone: 13044562206

Ground-based and Airborne Telescopes V, edited by Larry M. Stepp, Roberto Gilmozzi,

Helen J. Hall, Proc. of SPIE Vol. 9145, 91450B • ( 2014 SPIE

CCC code: $0277-786 X / 14 / \$ 18 \cdot$ doi: $10.1117 / 12.2056761$

Proc. of SPIE Vol. 9145 91450B-1 
studies, ${ }^{1}$ and some upgrades of the telescope were performed at that time. ${ }^{2}$ The RadioAstron project operates at a much higher frequency than the previous ionospheric project, so more care was needed in the refurbishment of both the optical and control systems. The Lebedev Physical Institute (LPI) of the Russian Academy of Sciences supplied a complete electronics suite to collect and store transmitted data.

This paper describes all upgrades to the 140ft for the GBES, beginning with a description of the antenna upgrades in Section 2, and followed by descriptions of the Cassegrain focus cabin infrastructure in Section 3, LPIsupplied equipment in Section 4, the Mark 5C data recorder in Section 5, software in Section 6, data collection and handling in Section 7, and a summary in Section 8.

\section{ANTENNA UPGRADES}

\subsection{Antenna Optics}

For the RadioAstron GBES, the 140ft antenna is operated with the receiver at the Cassegrain focus. This position is ideal due to the relatively high frequency used for downlinking RadioAstron data, as the 140ft design includes a deformable subreflector that can be adjusted in real time to compensate for gravitationally induced distortions of the primary mirror, which limit performance at short wavelengths. The upgrade included a refurbished deformable subreflector to ensure there was enough gain to downlink the data at Ku-band, even at the farthest distance of the satellite's highly eccentric orbit. It became apparent, however, that this deformable subreflector was not needed for the RadioAstron project given that the signal-to-noise ratio was sufficient without the use of the deformer.

\subsubsection{Deformable Subreflector}

The 140ft antenna is fitted with a deformable subreflector designed to compensate for the distortions in the primary mirror due to gravity. ${ }^{3}$ The deformable subreflector has not been used for many years because recent $140 \mathrm{ft}$ projects only used the prime focus receiver location. The RadioAstron project's higher frequencies (8.4 and $15 \mathrm{GHz}$ ) demanded that the Cassegrain focus be used. After the deformable subreflector was removed from storage, its surface was surveyed and set to the nominal hyperboloidal shape. A new actuator control system and new actuators, which were chosen to eliminate radio frequency interference (RFI) that was produced by brush-type motors on the earlier generation of actuator motors, were installed. As the new AC motors do not generate any RFI, they are suitable for mounting in the open air behind the subreflector without causing interference to the $140 \mathrm{ft}$ telescope or to any other site telescopes. The new control system, however, consists of a small microcontroller board and a custom solid state relay board that generates significant RFI and is therefore mounted on the back of the subreflector in an RFI-shielded box. The actuator motor control system is shown in Figure 2.

\subsubsection{Subreflector Positioning Upgrades}

The upgrades to the 140ft telescope optics included rebuilding the positioner and the deformable subreflector. The positioning system, which is used to select the receiver for use, consists of a focus mechanism and a rotation mount. This system was upgraded to modern brushless controllers and motors, which were interfaced to the control system for automatic positioning of the subreflector. Brushless motor controllers create a large amount of RFI due to the pulse width modulation, requiring that these controllers be placed in RFI-tight enclosures to prevent interference with observations at radio telescopes on site (see Figure 3).

\subsection{Cassegrain Focus Cabin Upgrades}

The 140ft has a large cabin located at the Cassegrain focus of the telescope. In addition to providing a place to mount the feed horns, this cabin provides space for the receiving system electronics and transmission equipment that sends signals to the Jansky Laboratory at NRAO's Green Bank site (hereafter referred to as the Jansky Lab). Upgrades made to the Cassegrain focus cabin to support the new RadioAstron instrumentation are discussed below. 


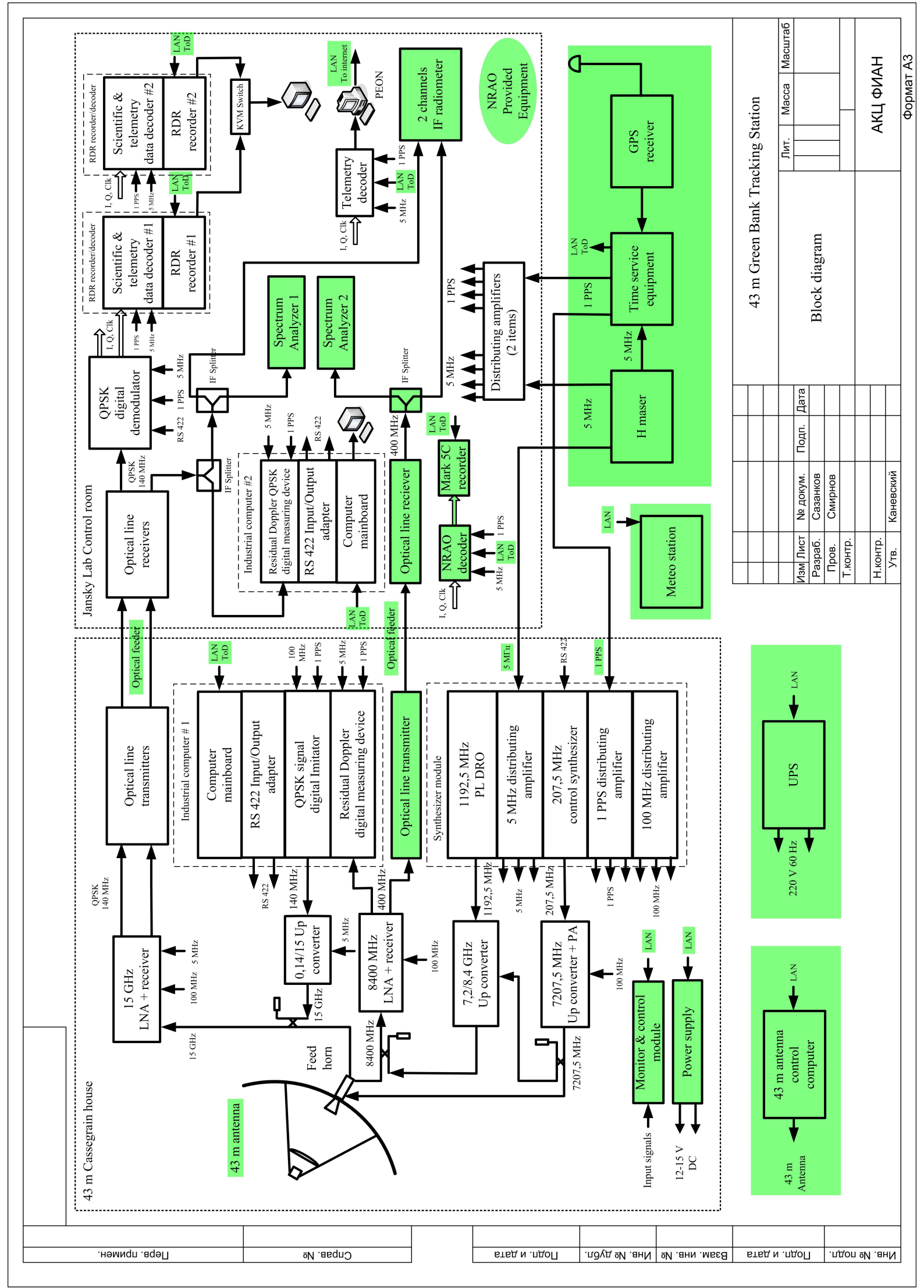

Figure 1. Block diagram of the Green Bank Earth Station, including both the LPI-supplied equipment and the NRAOsupplied equipment (shaded). 


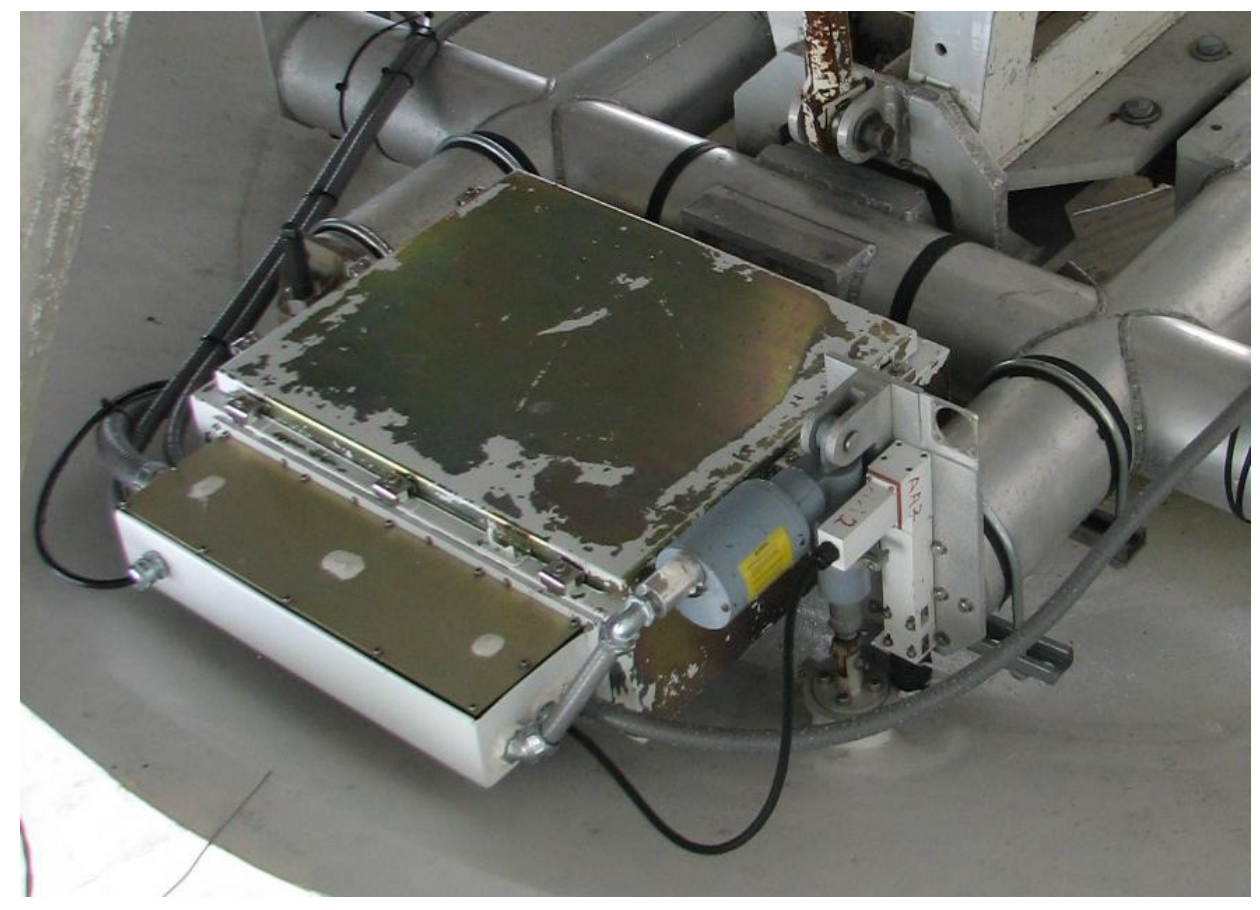

Figure 2. The actuator motor control system is contained in this RFI-tight box to suppress interference. An actuator is visible on the right.

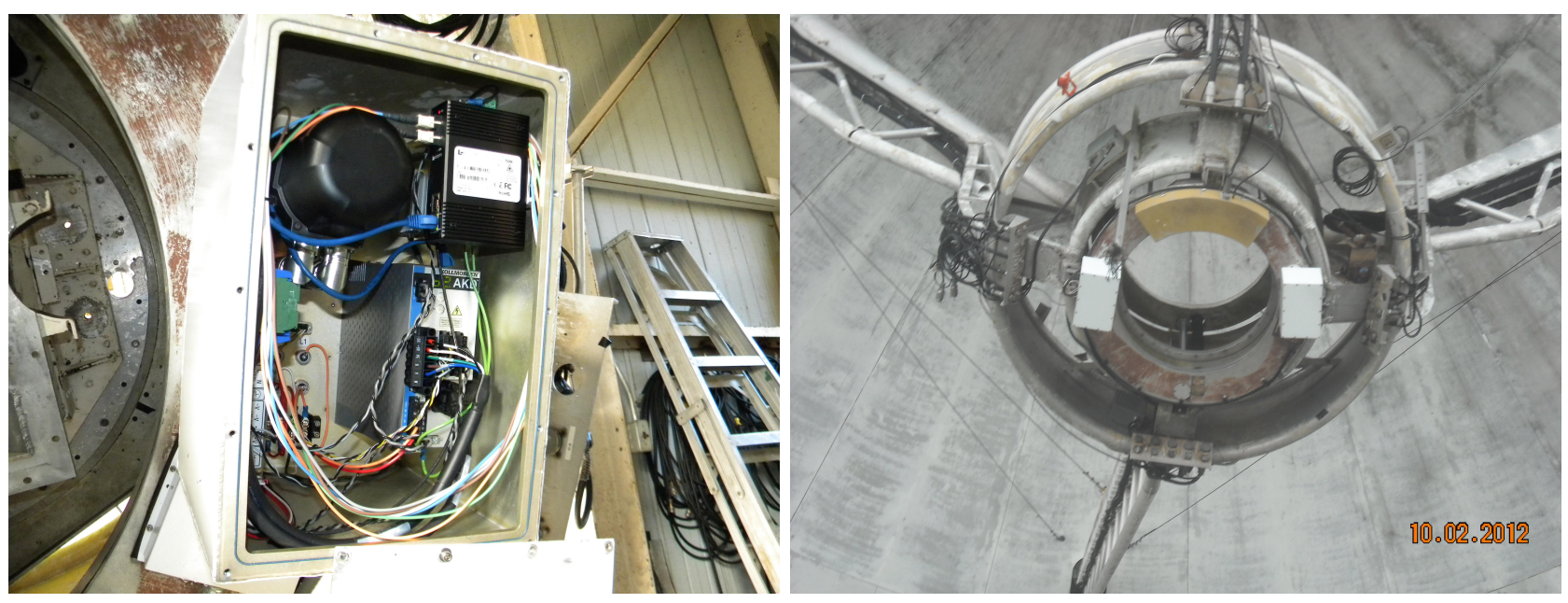

Figure 3. The motor and controller are contained in an RFI-tight box to suppress interference from the controller (left). Two RFI-tight boxes are mounted to the focus support ring (right). 


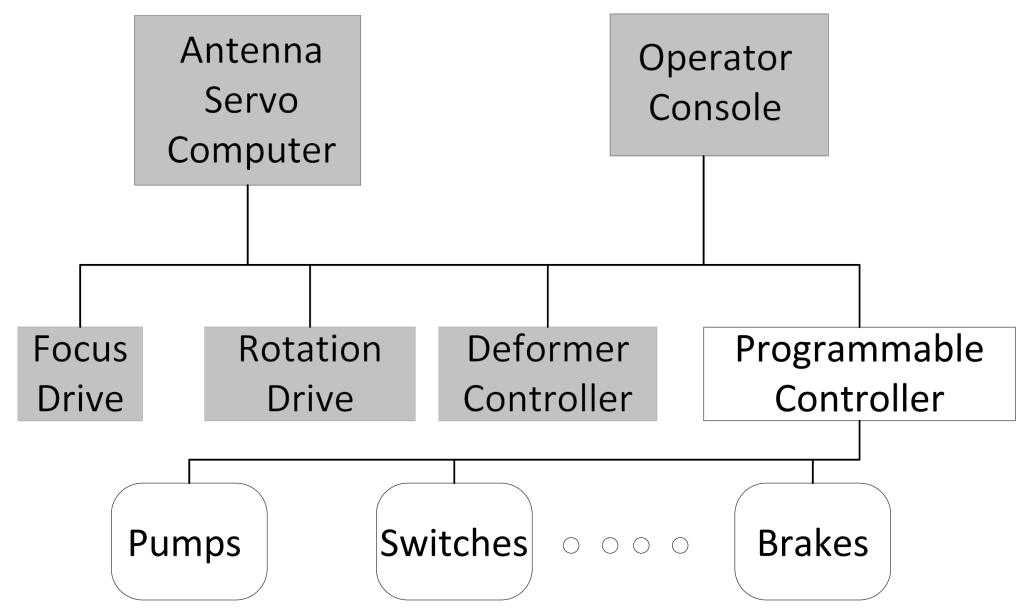

Figure 4. Antenna control system. Shaded blocks denote components upgraded for the RadioAstron project.

\subsubsection{Fibre Optics}

A 72 fibre single-mode cable was installed from the Jansky Lab to the 140ft telescope pedestal. Additional fibre cables were routed from the pedestal to the focus cabin, and are used to carry precision frequency references and timing signals, along with standard $1 \mathrm{~Gb} \mathrm{sec}^{-1}$ Ethernet signals. 12 multi-mode and 12 single-mode fibres were installed, with 9 spare multi-mode fibres and 3 spare single-mode fibres remaining after all RadioAstron equipment was connected.

\subsubsection{Temperature Control}

An upgraded temperature control system for the focus cabin was installed to keep the temperature of the electronics stable, thereby enabling the phase stability required for VLBI measurements. A new water chiller was installed, which, in combination with refurbished air handlers and resistance heaters, maintains the internal cabin temperature to within $\pm 2^{\circ} \mathrm{C}$ of that desired.

\subsubsection{Equipment Shielding}

An RFI-shielded rack houses all of the electronics for the receiving system. The shielded rack also contains an uninterruptible power supply that powers all of the guest receiving system electronics, which are discussed in Section 4 .

\subsection{Antenna Controls}

The antenna control system was redesigned and modernised in 2005. That incarnation of the system used an already aging system based on the Very Long Baseline Array (VLBA) control computer, which is now fully obsolete and is being retrofitted out of the VLBA. Before beginning RadioAstron downlinking, the control system was again modernised to eliminate that obsolete computer. Upgraded parts of the antenna control system are denoted by shaded blocks in Figure 4.

The servo computer hardware was upgraded to an Intel Core 2 Duo CPU with 3 GB of memory, and PCIe interface cards replaced the aging ISA bus cards. The operating system was upgraded to the Red Hat 6 Linux operating system including the Xenomai ${ }^{4}$ real time extensions for hard real time scheduling of the servo loops.

The servo system software and user interfaces were updated to control the subreflector deformer, the focus positioner, and the rotation positioner. An additional layer of software, the Antenna Manager, was developed to interface the servo system with the higher-level control system as discussed in Section 6.1, allowing simplified operations and user control of the position of all optical elements. A description of the movable optical elements is given in Section 2.1 while the controllers that position the optical elements are shown in the left panel of Figure 3. 


\section{CASSEGRAIN FOCUS CABIN INFRASTRUCTURE}

\subsection{Timing References}

A reference transmission system from the previous Orbiting Very Long Baseline Interferometry (OVLBI $\left.{ }^{5,6}\right)$ Earth station project was modified for the GBES. The system consists of three modules, the first an L102 VLBI LO Transmitter Module modified for the OVLBI project and designated the L102-OV. This module receives 10 and $100 \mathrm{MHz}$ from the Hydrogen maser reference approximately a metre away, phase-locks a $500 \mathrm{MHz}$ crystal oscillator to the $100 \mathrm{MHz}$ reference, combines the 10 and $500 \mathrm{MHz}$ references, and transmits them to the focus cabin via single-mode optical fibre. At the focus cabin is an L105 VLBI LO Receiver Module modified for the OVLBI project and designated the L105-OV, then modified again for RadioAstron and designated the L105-RA. This module takes the reference signal, separates out the 10 and $500 \mathrm{MHz}$ references, phase-locks a $500 \mathrm{MHz}$ crystal oscillator to the $500 \mathrm{MHz}$ reference, filters and re-synchronises the $10 \mathrm{MHz}$ reference to the crystal oscillator $500 \mathrm{MHz}$, phase-locks a $5 \mathrm{MHz}$ crystal oscillator and a $100 \mathrm{MHz}$ crystal oscillator to the resynchronised $10 \mathrm{MHz}$ reference and presents these references at SMA connectors on the rear panel of the module. The $500 \mathrm{MHz}$ reference is provided through a power divider to the rear panel and to a fibre transmitter on a single-mode fibre going back to the Jansky Lab, where it is sent to an L103 VLBI Round-Trip Phase Module modified for the OVLBI project and denoted the L103-OV. Here it is compared to the $500 \mathrm{MHz}$ reference from the maser and the round-trip phase is measured and logged.

\subsection{NRAO Ku-band Receiver}

It was necessary to have a receiver on the antenna for verification and adjustment of the antenna control software prior to the import and installation of the LPI-supplied equipment. A Ku-band receiver covering 14.5 to $15.35 \mathrm{GHz}$ from the OVLBI tracking station was repurposed and installed at the Cassegrain focus ring of the 140ft. Lab measurements show an uncooled receiver temperature of $\sim 300 \mathrm{~K}$. Although the feed horn used from the OVLBI project is not well matched to the optics of the 140ft, the spillover around the subreflector is cold sky and therefore does not adversely affect use of the receiver. A single channel downconverter was installed in the focus cabin and the 10-900 MHz IF was transported to power samplers in the antenna pedestal by coaxial cable for early antenna testing. The final installation has a dual channel downconverter in the focus cabin shielded rack and the IFs are transported to the Jansky Lab over new single-mode fibres.

\subsection{NRAO X-band Receiver}

After installation of the LPI-supplied receivers, additional information regarding the performance of the antenna control software was desired. The receiver temperatures of the downlink receivers were high enough that most celestial objects were invisible to the extent that precise pointing information was obtainable. An X-band receiver covering the 8.0 to $8.5 \mathrm{GHz}$ Space Research/Space-to-Earth/Deep Space allocation was constructed using repurposed parts and commercial, off-the-shelf (COTS) room temperature low-noise amplifiers. A feed horn from the 1986-87 outfitting of the Karl G. Jansky Very Large Array (VLA) used for Voyager flybys of Neptune and Uranus, which coincidentally, closely matches the optics of the 140ft, is being adapted for installation at the Cassegrain focus ring of the 140ft. To this horn is attached a waveguide ortho-mode transducer (OMT) from the same VLA outfitting, a waveguide adapter to fit the COTS amplifiers to the OMT, the COTS amplifiers, and a dual channel downconverter. The downconverted IF $(1.0-1.5 \mathrm{GHz})$ is transported to the Jansky Lab over single-mode fibres and can be connected to the total power detectors or any other available backend. System temperatures are in the $100 \mathrm{~K}$ range for this receiver, making it a powerful tool for further antenna testing and spacecraft downlinks.

\subsection{Total Power Detectors}

In parallel with the $15 \mathrm{GHz}$ data demodulator and the $8.4 \mathrm{GHz}$ Doppler measurement system are total power detectors that are used to measure signal levels from the LPI receiver systems. Power dividers in the focus cabin split the $15 \mathrm{GHz}$ and $8.4 \mathrm{GHz}$ signals to focus cabin use and to optical fibre feeds to the Jansky Lab. At the Jansky Lab the $15 \mathrm{GHz}$ feed is divided between a Doppler measurement module, a power detector and a spectrum analyser, and the $8.4 \mathrm{GHz}$ feed is divided between a power detector and a spectrum analyser. The 


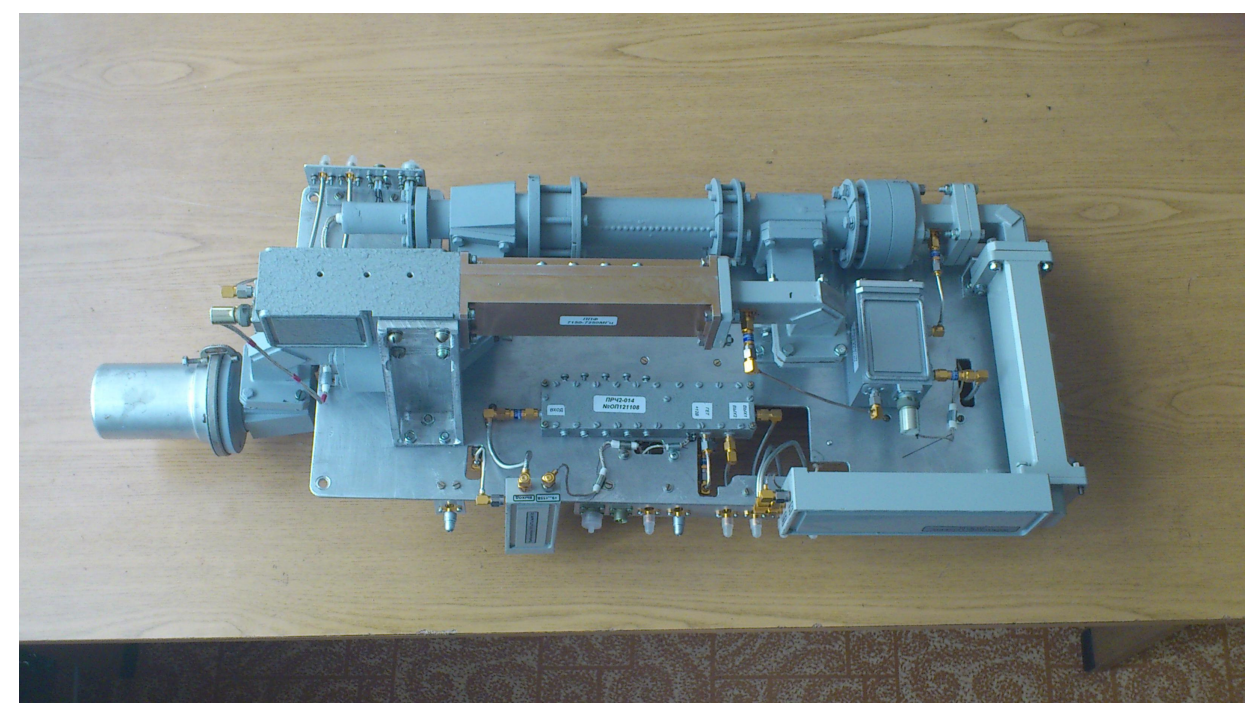

Figure 5. LPI-supplied front end.

spectrum analysers are remotely accessible via USB and their data are provided to the operator for monitoring signal quality during downlink sessions.

The power detector system is assembled from surplus components from other Green Bank telescopes. Following power division, each signal goes through a manually-adjusted variable attenuator set to optimise detector response, then to a Schottky diode detector followed by a DC-to- $20 \mathrm{~Hz}$ amplifier with a gain of 1000. The output of the amplifier is digitised to 24 bits by a USB data acquisition module and the data stream is used by the pointing software to optimise antenna pointing.

\section{LPI-SUPPLIED EQUIPMENT}

The LPI supplied a complete system to collect and store data downlinked from the RadioAstron satellite. Installed on the $140 \mathrm{ft}$ antenna is a conical feed horn connected by waveguide to a microwave unit with a $15 \mathrm{GHz}$ dual stage, low-noise downconverter, an $8.4 \mathrm{GHz}$ downconverter, and a $7.2 \mathrm{GHz}$ transmitter module. The downconverters and transmitter are coupled to the feed horn waveguide through the frequency-discriminating waveguide device shown in Figure 5. This prevents unit-to-unit interaction and minimises signal loss. The $15 \mathrm{GHz}$ downconverter supplies a $70 \mathrm{MHz}$ wide QPSK signal centred at $140 \mathrm{MHz}$ and the $8.4 \mathrm{GHz}$ downconverter supplies a $400 \mathrm{MHz}$ CW signal for Doppler tracking calculations.

An LPI computer, which is installed in an RFI-shielded rack in the focus cabin, performs the Doppler calculations, the transmission signal generation and the generation of a satellite imitator signal for testing the data handling system. Also installed in this rack are the fibre optic transmitter modules for sending the signals to the Jansky Lab.

At the Jansky Lab, the signal is received by the fibre optic receivers and sent to the QPSK demodulator. The output of the QPSK demodulator is sent to two identical decoder modules supplied by LPI, and to the NRAO supplied Mark 5C decoder described in Section 5. The decoder module strips off the telemetry headers and sends them to the telemetry processors, which then send the telemetry data back to the control centres for analysis. The science data is sent to the PC-based data recorders (RDR), where it is written to a removable hard disk. More information on the operation of the Earth station equipment can be found in the RadioAstron User Handbook. ${ }^{7}$

\section{MARK 5C DATA RECORDING SYSTEM}

In addition to the RDRs that are provided by the Astro Space Center (ASC), a standard Mark 5C VLBI data recorder was provided by NRAO. This recorder contains two removable disk packs that each contain 16 terabytes 
of disk space. A disk pack is used until full, and then the other disk pack is used while the first disk pack's data are transmitted to ASC over the internet, eliminating the need to ship disk packs around the world and avoiding unnecessary wear and tear on the disk packs.

In addition to the standard recorder, a custom decoder system was developed to decode the signal from the demodulator and format it for the Mark 5C. The decoder is based on the new ROACH Digital Backend developed by NRAO and the VLBI community to replace the aging analog backend systems that feed the older recording systems. The new digital backend receives the digital data stream from the demodulator and decodes it, stripping off the headers and sending them to a network socket. Once the headers are stripped off, the science data payload is removed and formatted into standard VLBI packets and streamed over the 10 Gb Ethernet link to the Mark 5C recorder.

\section{SOFTWARE}

\subsection{Antenna Control Software}

The prior 140ft antenna control software consisted of a twice-removed version of the VLBA station control software, which was used to control the $140 \mathrm{ft}$ during ionospheric experiments. As this version of the software lacked flexibility and was burdensome to operate, and because the GBT control software has been in use for nearly 15 years and has capabilities which exceed the requirements for the GBES, components of the GBT system were repurposed for GBES operation. Upgrading the antenna control software has minimised the amount of new software and software support, provided a system that support scientists were familiar with and had experience scripting, provided a system that operations already had experience with, and minimised the amount of operator training required.

The base infrastructure of the GBT control system is Ygor, ${ }^{8-10}$ a framework that provides monitoring and logging, control, and alarm/message management. Ygor has standard daemons to support various functions, including a message multiplexing daemon, a logging program that writes monitor data in FITS ${ }^{11}$ format files, and a coordinated logger that logs monitor data into FITS files during scans. Also included is a scan coordination daemon, which controls the start/stop timing of scans, selects devices that are to be controlled, and writes information about each device into a scan log for use by data analysis processes. Ygor based applications that use the framework are easily integrated with other parts of the system through a set of support libraries.

The antenna control system includes a rich set of primitives that allow trajectory specification to be stacked on different reference frames. Trajectory commands are specified as a list of parameterised basis functions that are interpolated for a given interval. Both linear and radial functions are supported. The linear function interpolates a position, velocity, and acceleration into a runtime command for a given interval. Other available basis functions can be used to specify Lissajous patterns, daisy petals, circles, ellipses, and spirals. Trajectories can be specified relative to a standard reference frame, or to a frame specified by an ephemeris or two-line element (TLE) orbit description. For example, while tracking an ephemeris or TLE orbit description, a circular motion centred upon the tracking position can be performed. This allows us to improve pointing accuracy by peaking up on the satellite without losing contact.

\subsection{User Interfaces}

User interfaces include the Astronomer's Integrated Desktop $\left(\right.$ Astrid $\left.^{12}\right) /$ turtle, which allow observation scripting for GBES automation, and a modified version of the GBT's Control Library for Engineers and Operators $\left(\mathrm{CLEO}^{13}\right)$, which is a large suite of GUI applications that are meant to be used by staff for high- and low-level monitoring, controlling, and debugging of all aspects of the GBT hardware. CLEO, written in TCL/Tk, sits on top of the underlying monitor and control system, which is written in $\mathrm{C}++$. The $140 \mathrm{ft}$ is now the third NRAO telescope that has used CLEO for these purposes. Both of these interfaces support multiple coordinated access from multiple locations, allowing support staff to quickly diagnose and resolve any technical issues from off-site locations.

To make use of the measurements of the wideband detector, we created a GUI application, "Convert Wideband," using the infrastructure of CLEO. In most telescope control systems, including the GBT, a combination of different programs are written for collecting the data from a backend, collating the measurements with the 
positions of the telescope, writing the results to disk, displaying for users some aspects of the data to provide integrity checking, providing messages and feedback to the user on the status of the instrumentation, performing the least-squares fit on pointing measurements, and applying in the antenna control system the determined pointing corrections. The CLEO "Convert Wideband" application was written to do all these things as we considered the wideband detector to be a very simple 'backend', and, unlike most radio telescopes, the scope of possibilities for what the above steps must do is very limited.

Due to the flexibility of the underlying monitor and control system, some of the major CLEO applications that were written for the GBT are also useful for the $140 \mathrm{ft}$ control system. A few needed minor changes, e.g., the antenna application was modified because the GBT uses an azimuth/elevation mount while the 140ft has an equatorial mount. Other applications, such as those that were designed for a specific GBT receiver, were removed from the $140 \mathrm{ft}$ version of CLEO.

\subsection{Operations Automation}

An important goal for the software system was to provide as much automation as possible. This was imperative as the same telescope operator monitors observations of the $140 \mathrm{ft}$, the GBT, the $45 \mathrm{ft}$, and the $20 \mathrm{~m}$ antenna. Automation reduces the workload on the operator, thereby reducing operating costs while maintaining reliability. To that end, we wrote a series of programs for downloading and processing of files that are needed for executing an observing session. This included the automatic downloading of VEX files (for controlling the data recorders), schedule files (which specified what runs are to be executed by the $140 \mathrm{ft}$ versus those to be executed by the Russian Earth station), and satellite trajectory files (also called ballistic files and which specify where in the sky to expect the Spektr-R satellite). Our software anticipates that each of these files can be updated on any time scale, up to a few hours before any downlink session.

We then created software that automatically converted these files into formats useful for the staff and the 140ft Monitor and Control system. This included: (1) converting the format of the satellite ballistic files into that of an Astrid ephemeris file; (2) converting schedule files into a flat database file that an Astrid scheduling block could then use to automatically start and stop observing sessions; (3) automatically copying files to the disks and computers expected by Russian hardware and software systems; and (4) automating the uploading of data and log files to LPI. Each of these processes were written as if they were real-time systems in that they automatically recover from a wide range of failure scenarios. Additionally, a series of watchdogs keep track of the progress made by the above software. The watchdogs keep local staff informed by e-mail whenever an expected product is late or missing.

We created an Astrid scheduling block that reads the schedule database and the Astrid ephemeris so as to prepare the antenna for tracking Spektr-R at the expected start time. By using external database and catalog files, the same Astrid script is run for every session - no editing is needed by the operator. The scheduling block executes multiple observing sessions without any interaction by the telescope operator. Each session usually lasts 1 hour in length, is separated by about 1 hour from the next, with usually two to four sessions scheduled back-to-back. Thus, the operator's only interaction with Astrid and the Monitor and Control system is to submit the scheduling block a few hours before a run and to stow the antenna any time after the end of a day's sequence of sessions.

One unique aspect of the Astrid scheduling block is the incorporation of a measurement of the pointing accuracy of the telescope while retaining the ability to simultaneously collect useful data. To do this, we have the telescope perform circles at the start of every observing session. The circles are centred at the expected position of the satellite and have a radius that takes the telescope through the expected half-power points of the telescope's beam. Since the error budget for our signals always allows us at least $6 \mathrm{~dB}$ of excess signalto-noise, we can successfully collect data even when we are executing this pointing maneuver. The pointing data are reduced automatically by the "Convert Wideband" CLEO application, described above, which then automatically corrects the telescope's pointing using the results of a 2-dimensional least-squares Gaussian fit.

\section{DATA COLLECTION AND HANDLING}

As the GBES's primary purpose is to collect data from the Spektr-R satellite, the telemetry data and log files that have been collected must be transmitted back to the satellite control centre and the science data sent to 
the correlator facility, which correlates data from all of the participating ground radio telescopes with the data downlinked from the RadioAstron satellite.

The log files and telemetry data volume are relatively small and can be directly transmitted to the ASC. The science data files, however, are very large. In order to successfully transmit these data, they are first copied from the real-time data acquisition computers onto a buffer computer, where they are stored until they can be sent over the internet to ASC for processing and distribution. Until very recently, the NRAO Green Bank site had a very limited capacity internet connection, which required data to be copied onto portable hard disks and sent to the NRAO Charlottesville site, where a more robust internet connection was used to transmit the large science data files. This procedure has been superseded by a faster internet connection in Green Bank.

The process of collecting data from each machine and sending it to the ASC has been automated by a collection of scripts that run on the buffer computer and check the progress of large data transfers, restarting processes when problems are encountered. The status of each file is maintained in a database, allowing support staff to verify files have been safely transmitted before erasing and reusing the data collection media.

\section{SUMMARY}

We have implemented the RadioAstron Green Bank Earth Station at the NRAO 140ft telescope in Green Bank, West Virginia. The 140ft antenna optics positioners were refurbished, with new motors and drives fitted to the secondary mirror positioning system, and the deformable subreflector was refurbished with a new digital controller and new actuators. The surface of the subreflector was measured and reset to its nominal hyperboloidal shape.

A new monitor and control system based on that of the GBT's Ygor and Astrid observation management system was developed, allowing the Spektr-R satellite to be tracked by executing a simple scheduling block. Tools were developed to automate antenna pointing during tracking. Data from the antenna control systems and logs are retained and delivered with the science and telemetry data for processing at the Astro Space Center of the Lebedev Physical Institute of the Russian Academy of Sciences and the mission control centre, Lavochkin Association.

NRAO provides infrastructure at the 140ft's Cassegrain focus cabin for use by the guest electronics systems, including timing references, Ethernet connectivity, and single-mode fibres to the remote Jansky Lab. An uncooled $\mathrm{Ku}$-band receiver is also available for testing, independent of the guest electronics. An X-band receiver is being adapted for pointing and antenna diagnostics, which covers the 8.0 to $8.5 \mathrm{GHz}$ Space Research/Spaceto-Earth/Deep Space allocation. Additional NRAO equipment supplied in the Jansky Lab for monitoring and controlling observations includes total power detector modules and remotely accessible spectrum analysers, allowing signal monitoring of both the 8.4 and $15 \mathrm{GHz}$ downlinks.

The LPI supplied a complete electronics suite to collect and store transmitted data, consisting of systems installed in both the 140ft focus cabin and the Janksy Lab. In the focus cabin, this includes a dual-frequency feed designed specifically for the 140ft, a dual-frequency receiver, an upconverter/transmitter for transmitting a reference tone to the satellite, and a control computer. At the other end of the optical fibre transmission system, the Jansky Lab houses the LPI-supplied demodulator, decoders, and data recorders. A second digital decoder module, based on the VLBA ROACH Digital Backend, was developed by NRAO's Socorro Electronics Division to decode data from the satellite and format it into standard VLBI packets, which are written to a standard Mark 5C VLBI data recorder. The duplicate system increases overall reliability and provides native formats to different correlators working with RadioAstron data.

Tests with the space radio telescope began mid-July 2013, while full operations began on September 5, 2013. For the operational period between July 2013 and Feb 28 2014, the GBES had 342 scheduled downlink sessions. Excluding the 6 sessions that were cancelled due to either ice accumulation on the 140ft or wind levels beyond the 140ft wind limits, the success rate of the GBES for the above operational period was $98 \%$. The GBES has covered 53\% of all RadioAstron downlink segments from September 2013 through May 2014. 


\section{ACKNOWLEDGMENTS}

We acknowledge the efforts of the many telescope mechanics and electronics division personnel who participated in the upgrade process, without whom the project would not have been possible. In addition, we thank Pete Chestnut and Dan Perera, who have contributed significantly to the successful downlink operations. The National Radio Astronomy Observatory is a facility of the National Science Foundation operated under cooperative agreement by Associated Universities, Inc. The RadioAstron project is led by the Astro Space Center of the Lebedev Physical Institute of the Russian Academy of Sciences and the Lavochkin Scientific and Production Association under a contract with the Russian Federal Space Agency, in collaboration with partner organizations in Russia and other countries.

\section{REFERENCES}

[1] Langston, G., "NRAO 43-m telescope operation at 170-1700 MHz: a Bi-Static Radar Collaboration," in [Highlights of Astronomy], Proceedings of the International Astronomical Union 2, 367-367 (8 2006).

[2] Ford, J. M., Langston, G., Shelton, J., and Weadon, T., "An extensible standards-based control system on a budget," in [Astronomical Telescopes and Instrumentation], 62740N-62740N, International Society for Optics and Photonics (2006).

[3] Wong, W., "The design of the deformable subreflector for the $140 \mathrm{ft}$. radio telescope," http://library.nrao.edu/public/memos/endir/EnDIR_110.pdf (1979).

[4] Various, "Xenomai: Getting started," http://www.xenomai.org/index.php/Getting_Started (2014).

[5] Hirabayashi, H., Hirosawa, H., Kobayashi, H., Murata, Y., Asaki, Y., Avruch, I. M., Edwards, P. G., Fomalont, E. B., Ichikawa, T., Kii, T., Okayasu, R., Wajima, K., Inoue, M., Kawaguchi, N., Chikada, Y., Bushimata, T., Fujisawa, K., Horiuchi, S., Kameno, S., Miyaji, T., Shibata, K. M., Shen, Z.-Q., Umemoto, T., Kasuga, T., Nakajima, J., Takahashi, Y., Enome, S., Morimoto, M., Ellis, J., Meier, D. L., Murphy, D. W., Preston, R. A., Smith, J. G., Wietfeldt, R. D., Benson, J. M., Claussen, M. J., Flatters, C., Moellenbrock, G. A., Romney, J. D., Ulvestad, J. S., Langston, G. I., Minter, A. H., D’Addario, L. R., Dewdney, P. E., Dougherty, S. M., Jauncey, D. L., Lovell, J. E. J., Tingay, S. J., Tzioumis, A. K., Taylor, A. R., Cannon, W. H., Gurvits, L. I., Schilizzi, R. T., Booth, R. S., and Popov, M. V., "The VLBI Space Observatory Programme and the Radio-Astronomical Satellite HALCA," PASJ 52, 955- (Dec. 2000).

[6] D'Addario, L., "OVLBI earth station memo series," www.gb.nrao.edu/ovlbi/memos.html (1990).

[7] RadioAstron Science and Technical Operations Group, "RadioAstron User Handbook," www.asc.rssi.ru/radioastron/documents/rauh/en/rauh.pdf (2014).

[8] Brandt, J. J., "Controlling the Green Bank Telescope," in [Advanced Telescope and Instrumentation Control Software II], Lewis, H., ed., Proc. SPIE 4009, 96-100 (2000).

[9] Clark, M. H., "Control software for the Green Bank Telescope," in [Telescope Control Systems III], Lewis, H., ed., Proc. SPIE 3351, 287-296 (1998).

[10] Ford, J. M. and Clark, M. H., "Ygor: An object oriented distributed telescope control system framework," in [Advanced Telescope and Instrumentation Control Software II], Lewis, H., ed., Proc. SPIE 4848, 12-22 (2002).

[11] Pence, W. D., Chiappetti, L., Page, C. G., Shaw, R. A., and Stobie, E., "Definition of the flexible image transport system (fits), version 3.0," A\&3A 524, A42 (2010).

[12] O'Neil, K., Shelton, A. L., Radziwill, N. M., and Prestage, R. M., "The Astronomer's Integrated Desktop: A unified suite of applications for scheduling-block based observing with the GBT," in [Astronomical Data Analysis Software and Systems XV], C. Gabriel, C. Arviset, D. P. and Solano, E., eds., ASP Conference Series 30 (2005).

[13] Maddalena, R. J., "The user interfaces for the NRAO-Green Bank Telescope," in [Advanced Telescope and Instrumentation Control Software II], Lewis, H., ed., Society of Photo-Optical Instrumentation Engineers (SPIE) Conference Series 4848, 316-327 (Dec. 2002). 